\title{
False vacuum decay in quantum mechanics and four dimen- sional scalar field theory
}

\author{
Maxim Bezuglov ${ }^{1,2, \star}$ \\ ${ }^{1}$ Bogoliubov Laboratory of Theoretical Physics, Joint Institute for Nuclear Research, Joliot-Curie 6, 141980 \\ Dubna, Moscow region, Russia \\ ${ }^{2}$ Moscow Institute of Physics and Technology (State University), 9 Institutskiy per., 141701 Dolgoprudny, \\ Moscow Region, Russian Federation
}

\begin{abstract}
When the Higgs boson was discovered in 2012 it was realized that electroweak vacuum may suffer a possible metastability on the Planck scale and can eventually decay. To understand this problem it is important to have reliable predictions for the vacuum decay rate within the framework of quantum field theory. For now, it can only be done at one loop level, which is apparently is not enough. The aim of this work is to develop a technique for the calculation of two and higher order radiative corrections to the false vacuum decay rate in the framework of four dimensional scalar quantum field theory and then apply it to the case of the Standard Model. To achieve this goal, we first start from the case of $d=1$ dimensional QFT i.e. quantum mechanics. We show that for some potentials two and three loop corrections can be very important and must be taken into account. Next, we use quantum mechanical example as a template for the general $d=4$ dimensional theory. In it we are concentrating on the calculations of bounce solution and corresponding Green function in so called thin wall approximation. The obtained Green function is then used as a main ingredient for the calculation of two loop radiative corrections to the false vacuum decay rate.
\end{abstract}

\section{Introduction}

As is well known, within the Standard model all particles acquire their masses through the Higgs mechanism. If we talk about it in a simplified way - the expectation value of Higgs field slides down to a minimum of some potential. At tree level this potential has one minimum. However, if we account for loop corrections, then at high energies it can form an additional deeper minimum [1-8]. Whether this second minimum arises or not depends on the masses of top quark and Higgs boson. With the known values of Higgs and top quark masses we get into the region in which this minimum is indeed formed. This means that the Higgs field can quantum tunnel from its current vacuum state into this second true vacuum, what will lead to an end of the known universe. The latter is not happening however. There are two possible solutions (not excluding each other) to this problem. The first is that there is some new physics connected with the Higgs sector that changes Higgs potential in such a way that all bad minimums there disappear. The second possibility is that the tunneling time to this minimum is much longer than the lifetime of the universe. Since we do not know anything about

^e-mail: bezuglov.ma@phystech.edu 

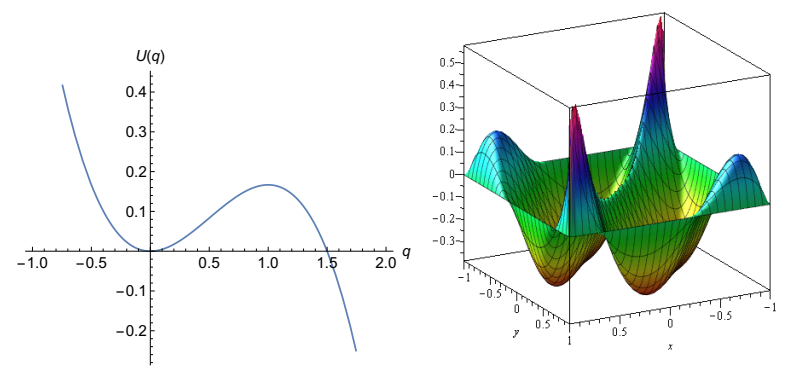

Figure 1. $U(q)=q^{2} / 2-\lambda q^{3} / 3$ on the left and corresponding instanton based Green function on the right

new physics, it makes sense to discuss the second option. Calculations show that the lifetime of the present false vacuum is large, but these calculations are carried out only at one loop ${ }^{1}$, which is apparently not enough. The aim of this work is to develop a technique for the calculation of two and higher order radiative corrections to the false vacuum decay rate in the framework of four dimensional scalar quantum field theory. To achieve this goal, we first start from the case of quantum mechanics and then we will use this example as a template for the general four dimensional theory.

\section{Quantum mechanics}

From the quantum mechanics we know that for a particle in a potential well there is a nonzero probability to jump out of it even if particle does not have enough energy for it in a classical sense. In a mathematical language it means that particles's energy acquires some imaginary part characterizing the tunneling probability. The mentioned imaginary part can be further extracted from the thermal partition function. However, it could not be obtained by means of perturbation theory at false vacuum. The main contribution to the imaginary part of the particles's energy is given by the factor $\exp \left(-S_{c}\right)$, where $-S_{c}$ is the euclidean action for the extremal trajectory minimizing euclidean action. Such a trajectory is called bounce or a instanton solution and to find it - it is enough to solve the usual equations of motion for the inverted potential. The latter corresponds to going to euclidean action by means of Wick time rotation.

Let's now discuss the corrections for the complex part of energy and consider the potential $U(q)=$ $q^{2} / 2-\lambda q^{3} / 3$, see left part of Figure 1 . The imaginary part of the ground state energy could be written as:

$$
\mathfrak{J} E_{0}(\lambda)=\sqrt{\frac{15 S_{c}}{2 \pi}} e^{-S_{c}}\left(1+\frac{I_{2}}{S_{c}}+\frac{I_{3}}{S_{c}^{2}}+O\left(\frac{1}{S_{c}^{3}}\right)\right)
$$

where $S_{c}=6 / 5 \lambda^{2}$ is the bounce action and $I_{2}, I_{3}$ are the two and three loop radiative corrections. To calculate two and three order corrections, we are following the method developed in [18-21] which is based on the Feynman diagram technique in coordinate representation. The main ingredient of this technique is the instanton based Green function. For the potential with cubic interaction we managed to find corresponding Green function analytically [22], the plot of which could be found on the righthand side of Figure 1.

\footnotetext{
${ }^{1}$ For tunneling rates calculations in Standard Model and its extensions see [9-17] and references therein.
} 


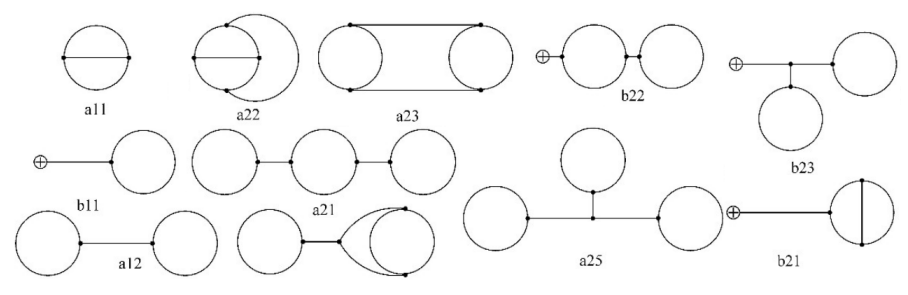

Figure 2. Two and tree loop Feynman diagrams for quantum mechanical case of the potential with the cubic term

The two-loop correction includes contributions from three Feynman diagrams, while there are already eight such diagrams at three-loops, see Figure 2. All these contributions were calculated numerically with the help of CUBA library [23] which uses the Monte Carlo technique. The results are $I_{2}=-1.4083307 \pm 7.85 * 10^{-6}$ and $I_{3}=-1.543 \pm 0.0045$. The numerical error in both cases is less than $1 \%$, which means that the calculations are sufficiently accurate, at least for qualitative conclusions. Both these contributions turn out to be of the order of unity and negative. Substituting these values into (1), we see that these corrections can significantly reduce the probability of decay in the case when $S_{c} \sim O(1)^{2}$. If $S_{c} \gg 1$, then these corrections do not have a qualitative effect on the decay.

More details and discussions about the quantum mechanical case can be found in our work [22] and references therein.

\section{False vacuum decay in $\mathrm{d}=4$ dimensional scalar quantum field theory}

Now, with the lessons learned in the case of quantum mechanics we may proceed with fourdimensional scalar field theory. For simplicity we will assume $\mathrm{O}(4)$ spherical symmetry of the bounce solution, so that the latter will depend only on one space coordinate. This reduces the problem of finding the bounce solution to that in one-dimensional case ${ }^{3}$, the only difference is the presence of the so-called dumping term which can be interpreted as some external force. On the the right part of the Figure 3 we plotted bounce solutions for the scalar potential of the Higgs type $\left(U(\varphi)=-m^{2} \varphi^{2} / 2 !+g \varphi^{3} / 3 !+\lambda \varphi^{4} / 4\right.$ !, see left part of the Figure 3$)$. Solutions were obtained numerically as this problem can not be solved analytically in the general case.

Nevertheless, some analytic approximation can still be done in the case when the true and false vacuums are practically degenerate, so that we can neglect the cubic term in the potential. This is the so-called thin-wall approximation. In this case the true vacuum is inside the bubble separated from the false vacuum by a wall, which thickness is much smaller than the size of the bubble.

To calculate higher order radiative corrections we again need to know an expression for Green function in instanton background. As usual, in order to find it, it is necessary to solve a second-order partial differential equation. The easiest way to solve it is to make use of spherical symmetry and expand the Green function in a series of spherical harmonics. For the potential discussed above, the coefficients of these series were calculated by Garbrecht and Millington within thing-wall approximation in [24]. In the case of zero harmonics their expression should be however corrected and is given

\footnotetext{
${ }^{2}$ Instead of the bounce action $\left(S_{c}\right)$, we can speak about the height of the barrier between the true and false vacuum, because these quantities are directly proportional to each other.

${ }^{3}$ Note, that the false vacuum decay in $\mathrm{d}=4$ dimensional case does not reduce entirely to one-dimensional, the similarity consists only in finding the bounce solution and the general principles on which the perturbation theory is constructed.
} 

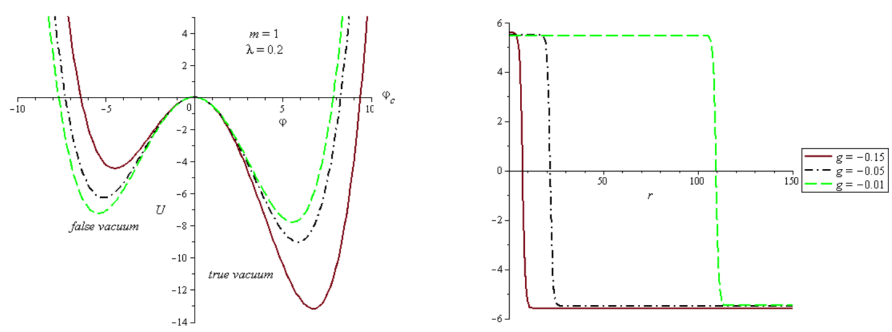

Figure 3. $U(\varphi)=-m^{2} \varphi^{2} / 2 !+g \varphi^{3} / 3 !+\lambda \varphi^{4} / 4$ ! on the left and the corresponding bounce solution on the right

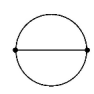

all

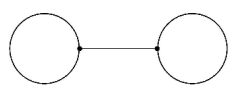

al2

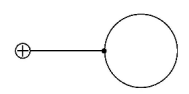

b11

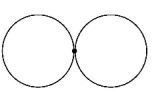

c11

Figure 4. Two loop Feynman diagrams for the $d=4$ dimensional scalar quantum field theory

by

$$
\begin{aligned}
G(x, y, 2)=\frac{g(x, y)}{4 \gamma}\left\{2-x y+\frac{|x-y|}{4}(11\right. & \left.-3 x y)+(x-y)^{2}\right\}+ \\
& +\frac{3}{32 \gamma}\left(1-x^{2}\right)\left(1-y^{2}\right)\left(\log g(x, y)-\frac{11}{3}\right)
\end{aligned}
$$

where: $g(x, y)=\frac{1-|x-y|-x y}{1+|x-y|-x y}, \gamma=\frac{\mu}{\sqrt{2}}, x=\tanh (\gamma(r-R)), y=\tanh \left(\gamma\left(r^{\prime}-R\right)\right)$. Here $r$ and $r^{\prime}$ is a radius in $\mathrm{O}(4)$ spherical coordinate system, $\mu^{2}=-m^{2}$ and $R=\frac{\sqrt{12 \lambda}}{q}$ is the bubble radius. Next, both one-loop functional determinant and higher order corrections could be calculated knowing this Green function. There is however one additional difficulty related to renormalization compared to quantum mechanical case. The ultraviolet divergences may be further regularized using momentum cutoff and so-called continuum approximation. Within the latter the summation over harmonics is rewritten as an integral over an almost continuous quantity. This technique and the method for finding the one-loop correction has been analyzed in detail in [24].

Now we can finally begin to study the higher order radiative corrections. The perturbation theory is constructed by the same principles as in quantum mechanics. The integrations are now however four-dimensional. Full two-loop correction acquire contributions from four diagrams, Figure 4. Here is an example of a contribution from "double bubble" diagram which is labelled "c11" on Figure 4:

$$
\begin{array}{r}
I_{c 11}=-\frac{\lambda \gamma^{3} R^{3}}{4 \pi^{2}}\left[\frac{1}{240}\left\{-135+10 \sqrt{3} \pi-\frac{9}{7} \pi^{2}+\left(4 \sqrt{3} \pi+45\left(2+\log \frac{\gamma^{2}}{\Lambda^{2}}\right)\right) \log \frac{\gamma^{2}}{\Lambda^{2}}\right\}-\right. \\
\left.-\frac{\Lambda^{2}}{48 \gamma^{2}}\left(\sqrt{3} \pi+9 \log \frac{\gamma^{2}}{\Lambda^{2}}\right)\right]
\end{array}
$$

where $\Lambda$ is ultraviolet cut-off regularization parameter.

\section{Conclusions}

In this work we used the method developed in [18-21] to calculate higher order radiative corrections to false vacuum decay rate in quantum mechanics and scalar quantum field theory. We show that in 
some cases these corrections may be significant and should be taken into account. At a moment we do not yet have a full two loop result in the case of scalar field theory. In this case we do have some technical difficulties related to the regularization of the sums over spherical harmonics in instanton Green functions. Still, we believe that this difficulties could be resolved and will be the subject of one of our future publications.

\section{Acknowledgements}

I would like to thank A.I. Onishchenko for interesting and stimulating discussions as well as for general assistance in writing this work. This work was supported by RFBR grants \# 17-02-00872-a.

\section{References}

[1] J. Elias-Miro, J. R. Espinosa, G. F. Giudice, G. Isidori, A. Riotto and A. Strumia, Phys. Lett. B 709, 222 (2012).

[2] G. Degrassi, S. Di Vita, J. Elias-Miro, J. R. Espinosa, G. F. Giudice, G. Isidori and A. Strumia, JHEP 1208, 098 (2012).

[3] F. Bezrukov, M. Y. Kalmykov, B. A. Kniehl and M. Shaposhnikov, JHEP 1210, 140 (2012).

[4] S. Alekhin, A. Djouadi and S. Moch, Phys. Lett. B 716, 214 (2012).

[5] I. Masina, Phys. Rev. D 87, no. 5, 053001 (2013).

[6] D. Buttazzo, G. Degrassi, P. P. Giardino, G. F. Giudice, F. Sala, A. Salvio and A. Strumia, JHEP 1312, 089 (2013).

[7] J. R. Espinosa, G. F. Giudice, E. Morgante, A. Riotto, L. Senatore, A. Strumia and N. Tetradis, JHEP 1509, 174 (2015).

[8] A. V. Bednyakov, B. A. Kniehl, A. F. Pikelner and O. L. Veretin, Phys. Rev. Lett. 115, no. 20, 201802 (2015).

[9] G. Isidori, G. Ridolfi and A. Strumia, Nucl. Phys. B 609, 387 (2001).

[10] Z. Lalak, M. Lewicki and P. Olszewski, JHEP 1405, 119 (2014).

[11] A. D. Plascencia and C. Tamarit, JHEP 1610, 099 (2016).

[12] M. Endo, T. Moroi, M. M. Nojiri and Y. Shoji, JHEP 1601, 031 (2016).

[13] Z. Lalak, M. Lewicki and P. Olszewski, Phys. Rev. D 94, no. 8, 085028 (2016).

[14] O. Czerwińska, Z. Lalak, M. Lewicki and P. Olszewski, JHEP 1610, 004 (2016).

[15] J. R. Espinosa, M. Garny, T. Konstandin and A. Riotto, Phys. Rev. D 95, no. 5, 056004 (2017).

[16] M. Endo, T. Moroi, M. M. Nojiri and Y. Shoji, Phys. Lett. B 771, 281 (2017).

[17] M. Endo, T. Moroi, M. M. Nojiri and Y. Shoji, arXiv:1704.03492 [hep-ph].

[18] A. A. Aleinikov and E. V. Shuryak, Yad. Fiz. 46, 122 (1987).

[19] S. Olejnik, Phys. Lett. B 221, 372 (1989).

[20] M. A. Escobar-Ruiz, E. Shuryak and A. V. Turbiner, Phys. Rev. D 92, no. 2, 025046 (2015)

Erratum: [Phys. Rev. D 92, no. 8, 089902 (2015)].

[21] M. A. Escobar-Ruiz, E. Shuryak and A. V. Turbiner, Phys. Rev. D 92, no. 2, 025047 (2015).

[22] M. A. Bezuglov and A. I. Onishchenko, Phys. Rev. D 96, no. 3, 036001 (2017).

[23] T. Hahn, CUBA: A Library for multidimensional numerical integration, Comput. Phys. Commun. 168, 78 (2005) [hep-ph/0404043].

[24] B.Garbrecht and P.Millington, Phys. Rev. D 91, 105021 (2015). 